Nicholas J. Prince

Katherine L. Brown

Teumzghi F. Mebrahtu

Roger C. Parslow

Mark J. Peters

\section{Weight-for-age distribution and case-mix adjusted outcomes of 14,307 paediatric intensive care admissions}

Received: 19 March 2014

Accepted: 19 June 2014

Published online: 18 July 2014

(C) The Author(s) 2014. This article is published with open access at Springerlink.com

Take home message: Children admitted to PICU are underweight compared to the UK reference population and weight-for-age at admission is an independent risk factor for mortality. Weight-for-age has a U-shaped relationship with mortality with the lowest risk in mild-to-moderately overweight children.

Part of this data was presented at the 24th Annual Meeting of the European Society of Paediatric and Neonatal Intensive Care, 12-15th June 2013, Rotterdam, the Netherlands; see Prince NJ, Brown K, Parslow RC and Peters MJ (Intensive Care Med 39(Suppl 1):S20, 2013).

Electronic supplementary material The online version of this article (doi:10.1007/s00134-014-3381-x) contains supplementary material, which is available to authorized users.

N. J. Prince $(\varangle) \cdot$ M. J. Peters

Respiratory Critical Care and Anaesthesia Unit, Institute of Child Health, University College London, London WC1N 1EH, UK e-mail: nprince@nhs.net

Tel.: +442078138213

K. L. Brown

Cardiac Critical Care Unit, Great Ormond Street Hospital for Children NHS Trust, London WC1N 3JH, UK
T. F. Mebrahtu · R. C. Parslow Division of Epidemiology and Biostatistics, Leeds Institute of Genetics, University of Leeds, Leeds LS2 9JT, UK

\section{J. Peters}

Paediatric and Neonatal Intensive Care Units, Great Ormond Street Hospital for Children NHS Trust, London WC1N 3JH, UK

Abstract Aims: To determine whether the paediatric intensive care (PIC) population weight distribution differs from the UK reference population and whether weight-for-age at admission is an independent risk factor for mortality. Meth-

ods: Admission weight-for-age standard deviation scores (SDS) were calculated for all PIC admissions (March 2003-December 2011) to Great Ormond Street Hospital: this is the number of standard deviations (SD) between a child's weight and the UK mean weight-for-age. Categorised into nine SDS groups, standardised mortality ratios (SMR) and logistic regression were used to assess the relationship between weight-for-age at admission and riskadjusted mortality. Results: Out of 12,458 admissions, mean weight-forage was 1.04 SD below the UK reference population mean $(p<0.0001)$. Based on 942 deaths, risk-adjusted mortality was lowest in those with mild-to-moderately raised weight-for-age (SDS 0.5-2.5) and highest in children with extreme under- or overweight (SDS $<-3.5$ and SDS $>+3.5$ ). Logistic regression indicated that age, gender, ethnicity and weight-for-age are independent risk factors for mortality. South Asian and 'other' ethnicities had significantly increased risk of death compared to children of white and black ethnic origin. Conclusion: The PIC population mean weight-for-age is significantly lower than the UK reference mean. The extremes of weight-for-age are overrepresented, especially underweight. Weight-for-age at admission is an independent risk factor for mortality. A U-shaped association between weight and risk-adjusted mortality exists, with the lowest risk of death in children who are mild-to-moderately overweight. Future studies should determine the impact of malnutrition on risk-adjusted mortality and investigate the aetiology of risk disparities with ethnicity.

Keywords Weight-for-age .

Malnutrition - Mortality ·

PICU weight distribution - Obesity . Underweight 


\section{Introduction}

Extremes of weight-for-age are common in children requiring intensive care and are likely to impact upon morbidity and mortality [1, 2]. Being underweight may indicate malnutrition, be associated with genetic syndromes, chronic conditions, or represent increased energy consumption [3, 4]. Protein-energy malnutrition is associated with increased mortality [5], can be complex to diagnose, and is often under recognised and underestimated $[6,7]$. Being overweight may reflect underlying disease or syndromes, limited mobility, or may simply represent increasing prevalence of obesity in the general population [8].

Being significantly underweight is associated with prolonged ICU stay and increased mortality [1, 9, 10]. However, published data on outcome in overweight children and adults is conflicting [11-14]. Broadly, adult intensive care unit (AICU) data suggest increased duration of mechanical ventilation and ICU stay but no clear association with mortality [15-17].

Weight-for-age may be an independent risk factor for mortality in critically ill neonates and children. In this study we tested the null hypotheses that the paediatric intensive care (PIC) population weight distribution does not differ from that of the general population and that weight-for-age at admission does not influence riskadjusted mortality.

\section{Methods}

We queried the prospectively collected Great Ormond Street Hospital (GOSH, London, UK) Paediatric Intensive Care Audit Network (PICANet) database to identify all children aged $0-18.0$ years admitted to the paediatric, cardiac and neonatal intensive care units between 1st March 2003 and 30th December 2011. GOSH is a tertiary and quaternary referral hospital for children with approximately 1,500-1,800 PIC admissions per year.

Demographic, admission and outcome information were recorded, including the paediatric index of mortality-2 (PIM2) score [18]. Children were recorded as dead if they died during the primary admission or within 30 days of discharge from PICU (including readmissions). Readmissions after more than 30 days were analysed as independent events. The Bloomsbury Research Ethics Committee confirmed that formal ethical approval was not required.

\section{Data preparation}

Up to chronological age 2 years, children born premature $(<37 / 40$ gestation) had their age corrected. Admission weight for each child was compared to UK population reference data by calculation of a weight-for-age standard deviation score (SDS); SDS is the number of standard deviations by which a weight value differs from the population mean. Standardising all weight values in this way allows comparison of children of differing age and gender. 'LMSgrowth' software [19] for Microsoft Excel was used, with UK growth reference data as per current Royal College of Paediatrics and Child Health guidelines [20-22]. Medical records of outliers were reviewed; children with anomalous or absent data were excluded.

Patients were categorised into nine groups according to their weight-for-age SDS (Table 1). A priori subgroup analyses were planned by age, gender and ethnicity. Four ethnic subgroups were chosen to avoid over-dividing the study cohort: white, black, South Asian and 'other'. The South Asian ethnic group was chosen since recent PICANet data has indicated poor outcomes across UK PICUs for this group of children [23, 24]. Age groups were chosen pragmatically: neonates including premature babies (group A), preschool infants and children (1 month4.9 years, group B) and school-age (5-18 years, group C).

\section{Data analysis}

The study population frequency distribution was compared to the UK reference population distribution using a one-sample $t$ test. A histogram of the study data was plotted against a representative distribution of the UK reference population.

The primary outcome was risk-adjusted mortality during PICU admission or within 30 days of discharge. Standardised mortality ratios (SMR) and $95 \%$ confidence interval $(95 \% \mathrm{CI})$ for each of the nine SDS groups were calculated [25] using PIM-2 scores. Multivariable statistical analysis was undertaken using logistic regression (STATA 12, StataCorp LP, Texas, USA) to assess the relationship between the main exposure variable (weightfor-age at admission), three covariates (age, gender, ethnicity) and the dependent variable (death). The logit form of PIM2 was used for mortality risk-adjustment and models were sequentially adjusted for each of the covariates. Likelihood ratio testing was used to select the best-fit model (Supplementary Appendix 2). A significance level of $5 \%$ (two-sided $p$ value 0.05 ) was used throughout. Results were expressed as odds ratios (OR) and $95 \%$ confidence intervals for risk of mortality.

\section{Results}

A total of 14,307 individual records were identified and 12,458 analysed: we excluded 102 records with absent or spurious weight data and 1,747 readmissions within 
Table 1 Study population characteristics and primary outcome data including details of distribution across demographic indices and weight-for-age at admission groups

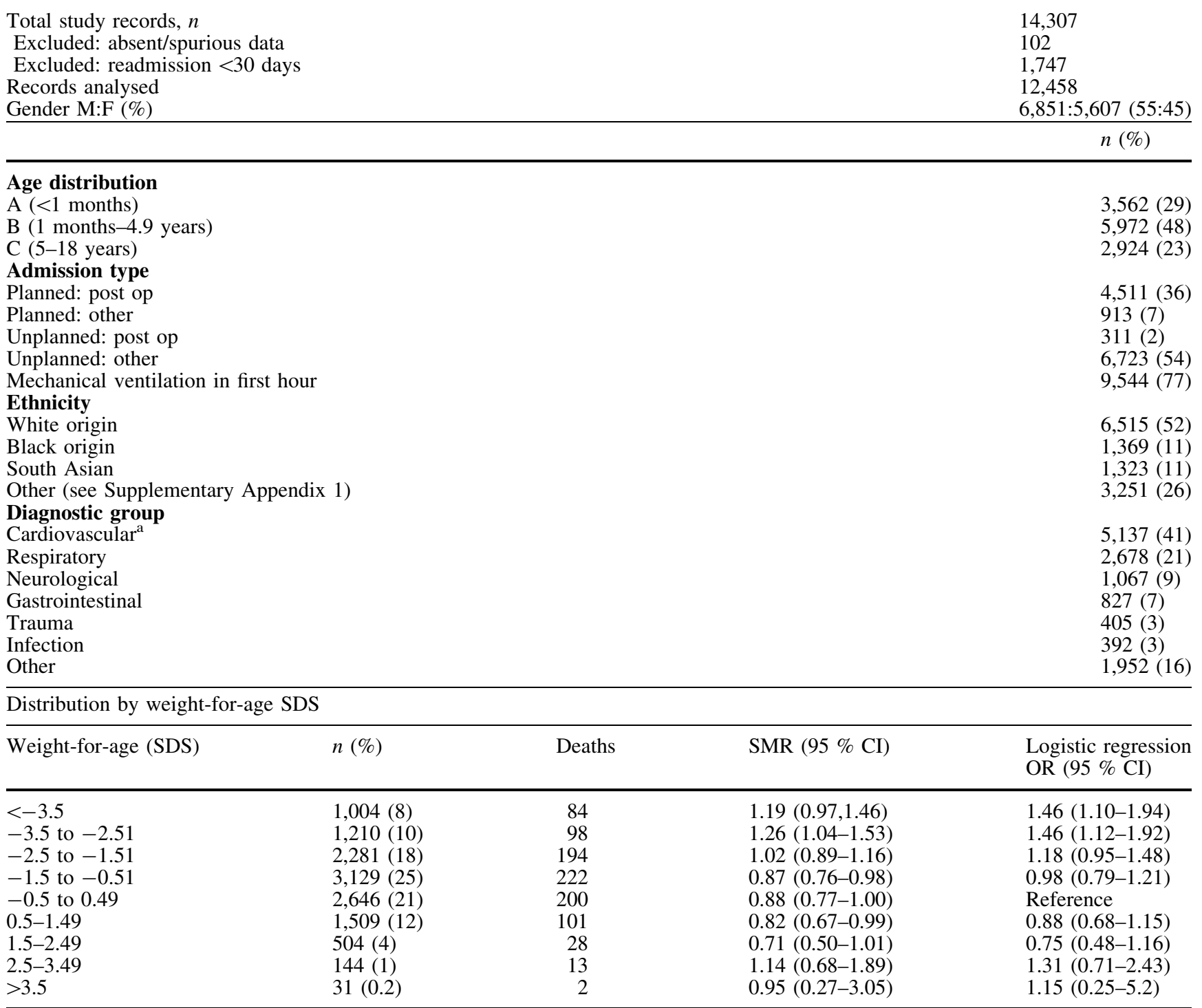

Study population weight distribution compared to UK reference population

Mean weight-for-age SDS (95\% CI)

Standard deviation

Median z-score weight-for-age
$-1.04(-1.07$ to -1.01$) p<0.0001^{*}$ 1.7 $-0.93$

Study population summary of deaths

Total deaths during study period

In PICU

$<30$ days post discharge

SMR for whole study population

Distribution of deaths, standardised mortality ratios (SMR) and odds ratio (OR) risk of death determined by logistic regression are provided by weight-for-age

*One-sample $t$ test comparison with population mean

a Post cardiac bypass $n(\%)$ : 2,859 (23) 
Fig. 1 Stacked histogram of study population admission Weight-for-age standard deviation score compared to UK reference population. Survivors and deaths are differentiated within the histogram. A representative UK reference population curve is superimposed for comparison. The mean weight-for-age at admission of the study population was 1.04 standard deviations less than that of the UK reference population (95\% CI -1.07 to -1.01 , $p<0.0001$ ), with an overrepresentation of outliers, especially extreme underweight. $S D S$ standard deviation score, $S D$ standard deviation
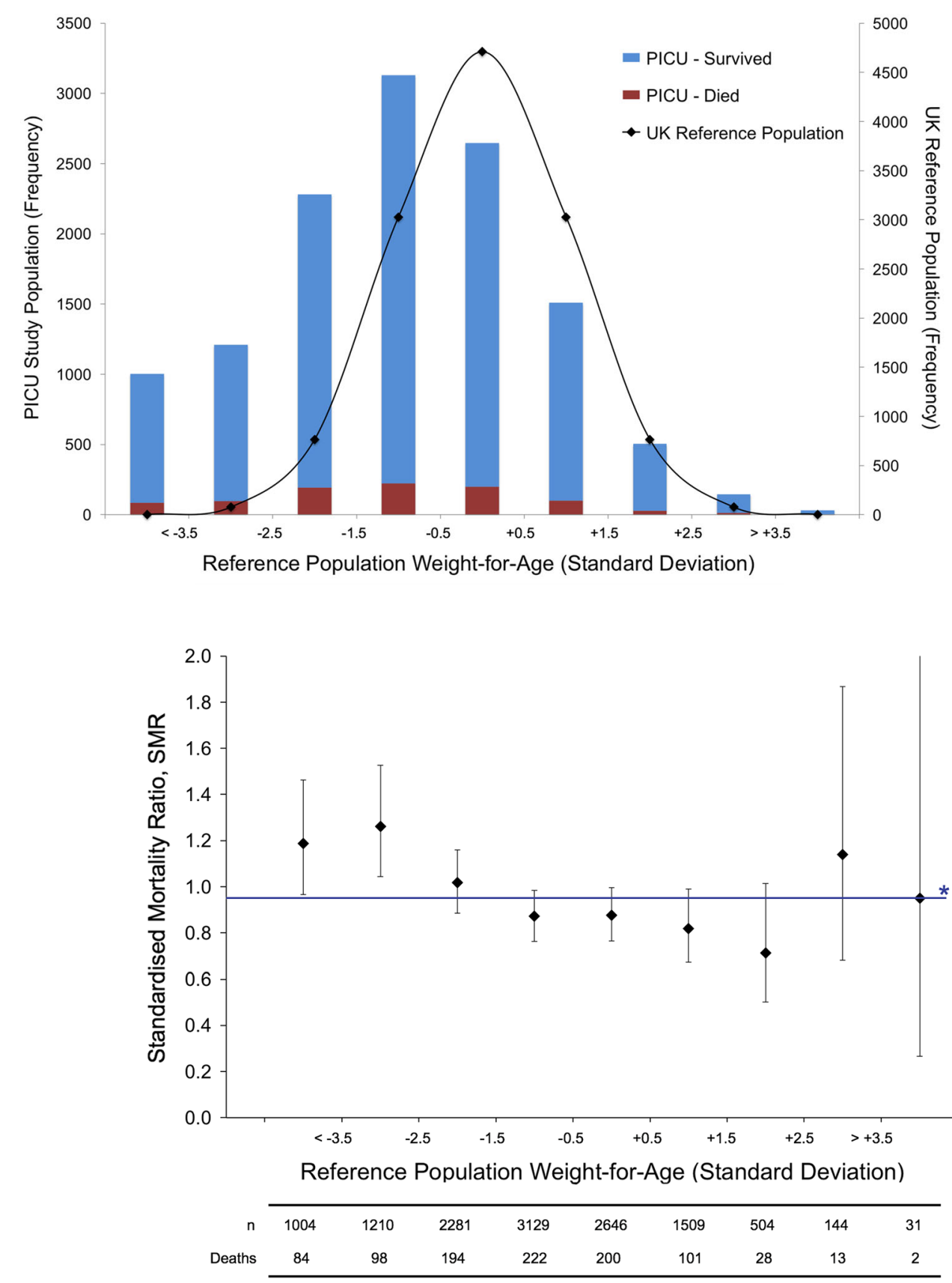

Fig. 2 Plot of standardised mortality ratios (SMRs) vs. weight-for-age at admission standard deviation scores, with $95 \%$ confidence intervals. Expected deaths are estimated by the sum of PIM2 scores for all patients in a group. SMRs are a ratio of observed to expected deaths. A 'U-shaped' association is indicated: highest risk of mortality occurs with extreme under- or overweight, which this study has shown to be over-represented in children admitted to PICU. The lowest risk of mortality occurred in children with a weight-for-age at admission between 0.5 and 2.5 standard deviations above the UK mean weight-for-age. Asterisk (*) indicates the overall SMR for the whole study cohort is displayed on the chart at 0.94

30 days. Baseline characteristics of the study population $p<0.0001) .18 \%$ and $1.4 \%$ of the study population are presented in Table 1. Fifty-five per cent were male, children were extremely under- or overweight (more than $52 \%$ were children of white origin, $11 \%$ black origin 2.5 standard deviations from UK mean weight-for-age), and $11 \%$ South Asian; $26 \%$ of children were therefore respectively (Fig. 1). grouped in the 'other' ethnicity category which included $4.9 \%$ Middle Eastern, $4 \%$ Asian, $3 \%$ mixed race, $7.5 \%$ ethnicity not provided and less than $1 \%$ each for all other Risk-adjusted mortality ethnicities (Supplementary Appendix 1).

The mean weight-for-age at admission of the study A total of 942 patients $(7.5 \%)$ died during the study population was 1.04 standard deviations less than that of period. The standardised mortality rate of the whole study the UK reference population ( $95 \%$ CI -1.07 to -1.01 , cohort was 0.94 [23]. The lowest risk of mortality 
occurred in children with a weight-for-age at admission between 0.5 and 2.5 standard deviations above the UK mean weight-for-age (Table 1, Fig. 2). The highest risk of mortality occurred in children with extreme under- or overweight (more than 2.5 standard deviations from the UK reference mean). Confidence intervals are wide for extreme overweight children because a relatively small proportion of the study population falls within these groups.

Trends in ORs for risk of death by weight-for-age at admission, determined by logistic regression, are comparable to SMR trends (Supplementary Appendix 2). The full logistic regression model, adjusted for covariates age, gender and ethnicity, was the best fit for the data; therefore the main exposure variable, weight-for-age at admission, and the covariates are all independent risk factors for mortality in children admitted to PICU.

\section{Subgroup analysis}

Age, gender and ethnic subgroups (Supplementary Appendix 3) have weight-for-age distributions which are comparable to the whole study cohort with two exceptions. First, young children $(<5$ years $)$ have markedly lower mean weight-for-age at admission than older children ( $<1$ months: $1.0,1$ months -5 years: 1.2 and 5-18 years: 0.6 standard deviations below the UK reference population mean, respectively). Second, South Asian children have a markedly lower mean weight-for-age compared to non-South Asians: 1.4 standard deviations below the UK mean vs. 1.0, 1.0 and 1.1 for children of white, black and 'other' ethnic origin, respectively. Exclusion of neonates did not substantially alter trends (Supplementary Appendix 5, Fig. 6g).

Odds ratios for risk of death within subgroups are presented in Fig. 3 and Supplementary Appendix 3.
Gender differences are non-significant $(p=0.06)$. Children 1 months -4.9 years have the lowest risk of dying whilst children of South Asian and 'other' ethnicity have significantly increased risk of death compared to those of white and black origin.

Subgroups have weight-for-age risk-adjusted mortality trends (Supplementary Appendices 3-6) comparable to the whole study cohort with two exceptions. First, in children older than 5 years of age, extremes of weightfor-age are not associated with increased risk of death (Supplementary Appendix 5). Second, in children of South Asian origin, moderate overweight does not appear to be associated with the lowest risk of mortality, though wide confidence intervals limit interpretation.

\section{Discussion}

We have demonstrated that the PIC population weight distribution at admission differs significantly from that of the UK reference population. Critically ill children have significantly lower weight-for-age than the UK average and also have an increased proportion at the extremes of weight-for-age, especially extreme underweight: 1.4 and $18 \%$ of the study population have a weight-for-age at admission more than 2.5 standard deviations above and below the UK population mean, respectively, rather than the expected $0.6 \%$ for each.

Multivariable logistic regression analysis demonstrated that weight-for-age at admission is an independent risk factor for mortality, as are age, gender and ethnicity. We observed a U-shaped association between weight-forage at admission and risk-adjusted mortality. U-shaped interactions occur between many biological risk factors and outcome [26, 27], but it is of particular interest that the lowest risk of death is associated with mild-to-
Fig. 3 Plot of odds ratio (OR) risk of death comparing categories within subgroups, determined by multivariable logistic regression, with $95 \%$ confidence intervals. The logit form of PIM2 was used for mortality risk adjustment with further sequential adjustment of the model for each covariate (gender, age and ethnicity). One category in each subgroup is used as the reference $(\mathrm{OR}=1)$ (see also Supplementary Appendix 3, Table 2). Age group: $A \leq 1$ month, $B=1$ month -4.9 years, $C=5-16$ years. Wh white, $B l$ black, $S A$ South Asian, $O$ other

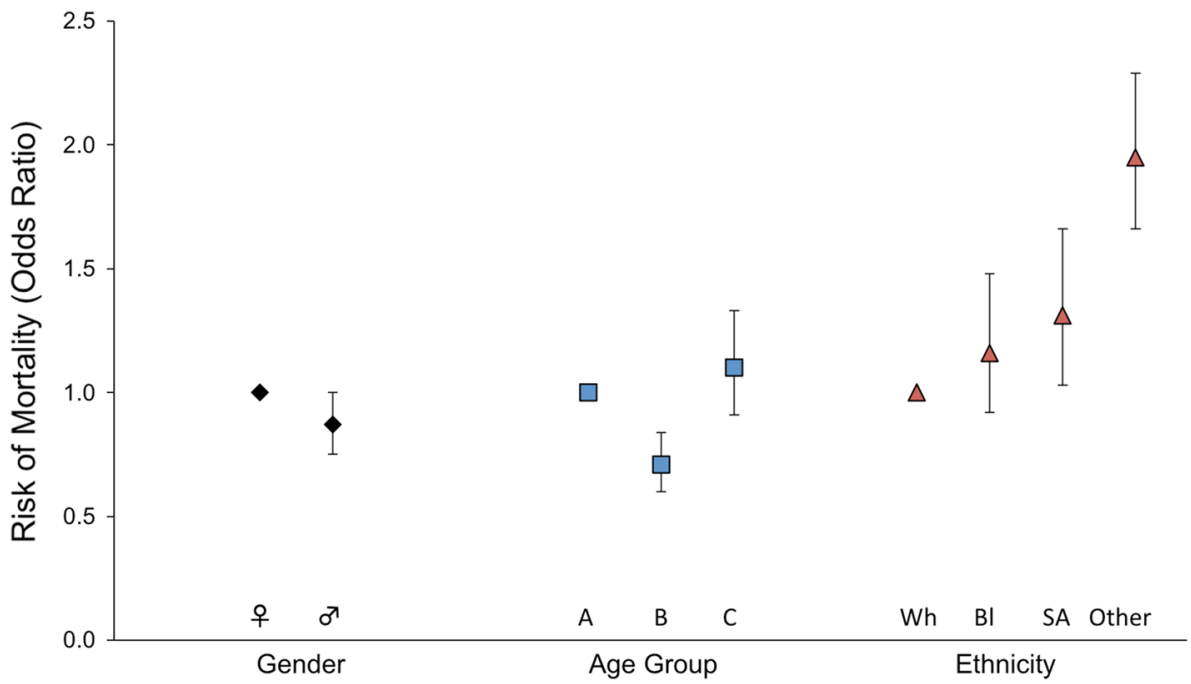


moderately raised, rather than mean, weight-for-age. Our data demonstrates that extreme under- and overweight is strongly associated with increased risk of death (Supplementary Appendix 5) and that these high-risk groups of children are markedly over-represented in the PIC population.

The associations we have observed could be unique to GOSH; our PICU differs from many others by providing very specialist services with a larger proportion of highrisk cases. The study mortality rate $(7.5 \%)$ is markedly higher than the UK average during the same period (4.6\%) whilst the risk-adjusted SMR is similar to other centres. However, comparable published data suggest that the results of this study may be generalizable to other centres [4]. Our findings are consistent with Numa et al. [1] who analysed 6,316 consecutive PICU admissions in Australia: a U-shaped relationship was seen with the lowest risk of death occurring at the 75th centile weightfor-age (equivalent to SDS +1.15). A similar relationship ('the obesity paradox') has been reported in adults with heart failure: lowest risk of mortality associated with mild-to-moderately overweight patients [28-30]. The proportion of underweight children in our cohort is very similar to two previous studies spanning 30 years. In the Netherlands, admission weight-for-age has been shown to be 0.3-0.9 SDS lower than the national average and 9-24 \% of patients are acutely malnourished at admission, dependent on age [4]. A US study in 1982 found $19 \%$ of critically ill children had acute protein-energy malnutrition at admission [5]. Given the specialist services provided at GOSH, these results should be replicated at other UK centres to confirm whether they are indeed generalizable.

In this study height measurements were not available and the study was not designed to take into account change in weight or nutritional status by PICU discharge or death. We cannot assume that underweight per se implies malnutrition. A recent study of admission, discharge and follow-up weight-for-age alongside anthropometric measurements found $24 \%$ of children were undernourished at admission; this increased by discharge but recovered to baseline at 6 months [4]. Their proportion of underweight children at admission is comparable both to historical data [5] and our own.

Syndromes may be associated with under- or overweight and several specific growth charts have been developed. We were unable to extract information regarding genetic syndromes but consider the effect on our results to be small. Trisomy 21 (T21) is the most frequent chromosomal abnormality and accounts for just $3.8 \%$ of PICU admissions [31]. Specific T21 growth chart data excluded children with known additional pathology and therefore may not be representative of children admitted to PICU [32].

We found a significantly increased risk of mortality in children of South Asian and 'other' ethnicity [24].
A South Asian phenotype of central adiposity, insulin resistance and larger, possibly dysfunctional, adipocytes has been described [33]. Published data suggests this may be present from childhood [34] or even in utero: cord leptin, a biomarker of body fat mass at birth, has been found to be higher in South Asian babies compared to white Europeans [35]. Why and how ethnicity is associated with differing risk-adjusted PIC mortality warrants further investigation. Aetiology is likely to be complex including, but not limited to, biological polymorphisms, socio-economic status, access to and equity of healthcare provision, lifestyle, diet and varying end-of-life care decisions according to ethnicity and culture $[36,37]$. UK reference population anthropometric data largely excludes children of non-white ethnic origin "due to known difference in growth" [22], limiting our ability to interpret weight-for-age in children of non-white ethnicity (Supplementary Appendix 3).

This study benefits from a large data set, which was collected prospectively and is externally validated. All admissions were included with only two pragmatic exclusions: to remove spurious data and to reduce bias from short-timescale readmissions of the same patient whilst including deaths within 30 days of discharge.

We accept that this study has a number of weaknesses. PICU admission weight often relies on weight measured at admission to the referring hospital or ward where most children are weighed as a routine. We acknowledge that some recorded values may be estimates and this reflects the reality of PICU practice; consistent with the approach taken in comparable recent studies [1], we believe any resultant error will be random and offset by our large data set. Subgroup analysis of elective admission, which might be expected to all have accurate admission weight, demonstrates similar trends to that shown in the whole study (Supplementary Appendix 7). UK reference anthropometric data will become increasingly outdated with time, especially with trends to increasing obesity [8]. Exclusion of non-white children means the data will not be representative of an increasingly diversified UK population.

This study has implications for clinicians and researchers alike. It defines the UK PIC population admission weight-for-age distribution, demonstrates weight to be a prognostic factor which should be considered at the bedside when assessing critically ill children and outlines variation with ethnicity. These results indicate that further work to fully understand the impact of weight-for-age and nutrition on outcome would be valuable. Weight was not considered during the development of PIM and PIM2; further work here may have some merit. One author has shown that including a weight variable in the PIM2 model leads to a small but statistically significant increase in the area under a receiver operating characteristic curve and considers how this may impact upon discriminatory predictive 
performance [1]. However, the benefits of adding variables must be balanced against the risk of complicating the tool and reducing accessibility and ease of use.

\section{Conclusion}

The PIC population weight distribution differs significantly from the UK reference population. The mean is significantly lower and there is an over-representation of patients at the extremes of weight-for-age; especially extreme underweight.

This study has shown that weight-for-age at admission is an independent risk factor for mortality. There is a U-shaped association between weight-for-age and riskadjusted mortality, with the lowest risk of death in children who are mild-to-moderately overweight. The overrepresented extremes of weight-for-age are associated with the highest risk-adjusted mortality. Future studies should determine the impact of malnutrition on riskadjusted mortality and investigate the aetiology of risk disparities with ethnicity.

Acknowledgments The authors would like to thank Peter Forbes, Royal Literary Fund Writing Fellow, for his advice and guidance during manuscript preparation. This work was undertaken at Great Ormond Street Hospital/UCL Institute of Child Health, which received a proportion of funding from the Department of Health's NIHR Biomedical Research Centre's funding scheme. Dr Peters receives higher education funding executive support as a clinical senior lecturer. Mr Mebrahtu is funded by The Hall Dorman Family $\mathrm{PhD}$ Scholarship.

Conflicts of interest No conflicts of interest to declare.

Open Access This article is distributed under the terms of the Creative Commons Attribution Noncommercial License which permits any noncommercial use, distribution, and reproduction in any medium, provided the original author(s) and the source are credited.

\section{References}

1. Numa A, McAweeney J, Williams G, Awad J, Ravindranathan H (2011) Extremes of weight centile are associated with increased risk of mortality in pediatric intensive care. Crit Care 15(2):R106

2. Reilly JJ, Methven E, McDowell ZC, Hacking B, Alexander D, Stewart L, Kelnar CJH (2003) Health consequences of obesity. Arch Dis Child 88(9):748-752

3. Mehta NM, Duggan CP (2009) Nutritional deficiencies during critical illness. Pediatr Clin North Am 56(5):1143-1160

4. Hulst J, Joosten K, Zimmermann L, Hop W, van Buuren S, Buller H, Tibboel D, van Goudoever J (2004) Malnutrition in critically ill children: from admission to 6 months after discharge. Clin Nutri 23(2):223-232

5. Pollack MM, Wiley JS, Kanter R, Holbrook PR (1982) Malnutrition in critical ill infants and children. JPEN J Parenter Enteral Nutr 6(1):20-24

6. Verhoeven JJ, Hazelzet JA, van der Voort E, Joosten KF (1998)

Comparison of measured and predicted energy expenditure in mechanically ventilated children. Intensive Care Med 24(5):464-468

7. Hudson LD, Cumby C, Klaber RE, Nicholls DE, Winyard PJ, Viner RM (2013) Low levels of knowledge on the assessment of underweight in children and adolescents among middle-grade doctors in England and Wales. Arch Dis Child 98(4):309-311
8. Branca F, Nikogosian H, Lobstein T (2007) The challenge of obesity in the WHO European Region and the strategies for response. World Health Organisation, Europe. Available at http://www.euro.who.int/_data/assets/ pdf_file/0010/74746/E90711.pdf. Accessed July 2013

9. Kielmann AAA, McCord CC (1978) Weight-for-age as an index of risk of death in children. Lancet 1(8076): 1247-1250

10. Goday PS (2008) Does admission weight influence mortality and morbidity in the pediatric intensive care unit (PICU)? JPEN J Parenter Enteral Nutr 32(3):316-317

11. Srinivasan V, Nadkarni VM, Helfaer MA, Carey SM, Berg RA (2010) Childhood obesity and survival after inhospital pediatric cardiopulmonary resuscitation. Pediatrics 125(3):e481e488

12. Goday PS, Vermilyea S, Kuhn EM, Otto MM, Mikhailov TA (2009) Admission anthropometric measures and outcomes in the pediatric intensive care unit (PICU). JPEN J Parenter Enteral Nutr 33:237-238

13. Goh VL, Wakeham MK, Brazauskas R, Mikhailov TA, Goday PS (2013) Obesity is not associated with increased mortality and morbidity in critically ill children. JPEN J Parenter Enteral Nutr 37(1):102-108
14. Oei G, Yee N, Henderson A, Noriega D (2011) Impact of obesity on morbidity and mortality in the pediatric intensive care unit. Crit Care Med 39:12 (suppl)

15. Akinnusi ME, Pineda LA, El Solh AA (2008) Effect of obesity on intensive care morbidity and mortality: a metaanalysis. Crit Care Med 36(1):151-158

16. Oliveros H, Villamor E (2008) Obesity and mortality in critically ill adults: a systematic review and meta-analysis. Obesity 16(3):515-521

17. Hogue CW, Stearns JD, Colantuoni E, Robinson KA, Stierer T, Mitter N (2009) The impact of obesity on outcomes after critical illness: a metaanalysis. Intensive Care Med 35(7):1152-1170

18. Slater AA, Shann FF, Pearson GG (2003) PIM2: a revised version of the paediatric index of mortality. Intensive Care Med 29(2):278-285

19. Pan H, Cole TJ (2011) LMSgrowth, a Microsoft Excel add-into access growth references based on the LMS method. Version 2.76. Available via http://www.healthforallchildren.co.uk. Accessed Oct 2012

20. RCPCH (2012) UK-WHO growth charts. Available at http://www. rcpch.ac.uk/growthcharts. Accessed 20 Nov 2012

21. Freeman JV, Cole TJ, Chinn S, Jones PRM, White EM, Preece MA (1995) Cross sectional stature and weight reference curves for the UK, 1990. Arch Dis Child 73:17-24 
22. WHO (2013) The WHO child growth standards. Available at http://www.who.int/childgrowth/ standards/en/ Accessed 10 Jan 2013

23. Draper E, Hobson R, Lamming C, McShane P, Norman L, Parslow R, Skinner S (2011) Annual report of the paediatric intensive care audit network. Available via http://www.picanet. org.uk/documentation.html. Accessed Oct 2012

24. Parslow RC, Tasker RC, Draper ES, Parry GJ, Jones S, Chater T, Thiru K, McKinney PA (2009) Epidemiology of critically ill children in England and Wales: incidence, mortality, deprivation and ethnicity. Arch Dis Child 94(3):210-215

25. Lowry R (2013) VassarStats: website for statistical computation. Available at http://vassarstats.net. Accessed 20 Nov 2012

26. Eastwood G, Bellomo R, Bailey M, Taori G, Pilcher D, Young P, Beasley R (2011) Arterial oxygen tension and mortality in mechanically ventilated patients. Intensive Care Med 38(1):91-98

27. Young PJ, Saxena M, Beasley R et al (2012) Early peak temperature and mortality in critically ill patients with or without infection. Intensive Care Med. doi:10.1007/s00134-012-2478-3
28. Habbu A, Lakkis NM, Dokainish H (2006) The obesity paradox: fact or fiction? Am J Card 98(7):944-948

29. Arabi YM, Dara SI, Tamim HM, Rishu AJ, Bouchama A, Khedr MK, Feinstein D, Parrillo J, Wood KE, Keenan SP, Zanotti S, Martinka G, Kumar A, Kumar A (2013) Clinical characteristics, sepsis interventions and outcomes in the obese patients with septic shock: an international multicenter cohort study. Crit Care 17(2):R72

30. Pickkers P, de Keizer N, Dusseljess J, Weerhejm D, van der Hoeven JG, Peek $\mathrm{N}$ (2013) Body mass index is associated with hospital mortality in critically ill patients. Crit Care Med 41(8):1878-1883

31. Tibby SMS, Durward AA, Goh CTC, Thorburn KK, Morris KK, Broadhead MM, Peters MJ (2012) Clinical course and outcome for critically ill children with Down syndrome: a retrospective cohort study. Intensive Care Med 38(8):1365-1371

32. Styles ME, Cole TJ, Dennis J, Preece MA (2002) New cross sectional stature, weight, and head circumference references for Down's syndrome in the UK and Republic of Ireland. Arch Dis Child 87(2):104-108
33. Chandalia M, Lin P, Seenivasan T, Livingston EH, Snell PG, Grundy SM, Abate N (2007) Insulin resistance and body fat distribution in South Asian men compared to caucasian men. PLoS One 2(8): $\mathrm{e} 812$

34. Ehtisham S, Crabtree N, Clark P, Shaw N, Barrett T (2005) Ethnic differences in insulin resistance and body composition in United Kingdom adolescents. J Clin Endocrin Meta 90(7):3963-3969

35. West J, Wright J, Fairley L, Sattar N, Whincup P, Lawlor DA (2014) Do ethnic differences in cord blood leptin levels differ by birthweight category? findings from the born in Bradford cohort study. Int $\mathrm{J}$ Epidemiol 43(1):249-254

36. Devictor DJ, Latour JM (2011) Forgoing life support: how the decision is made in European pediatric intensive care units. Intensive Care Med 37(11):1881-1887

37. Sprung CL, Maia P, Bulow $\mathrm{HH}$ et al (2007) The importance of religious affiliation and culture on end-of-life decisions in European intensive care units. Intensive Care Med 33(10):1732-1739 